Майре ОЯВЕЭР, Ю. СООЯРВ

\title{
АНАЛИЗ ПРОЦЕССОВ ПРЕОБРАЗОВАНИЯ ЭНЕРГИИ В ИНДУКТИВНО-КЛЮЧЕВОМ СГЛАЖИВАЮЩЕМ ФИЛЬТРЕ
}

\author{
(Представил Н. Эпик)
}

В Институте термофизики и электрофизики АН ӘССР разработаны новые схемы индуктивно-ключевых сглаживающих фильтроз $\left[{ }^{1,2}\right]$, в которых путем ритмичного изменения числа витков обмотки дросселя уменьшается размах пульсаций или подавляются самые низкие из высших гармоник в выходном токе.

В данной статье выводится выражение для определения формы тока в индуктивно-ключевом сглаживающем фильтре (рис. 1) при конечном значении индуктивности дросселя и анализируются процессы преобразования энергии в схеме.

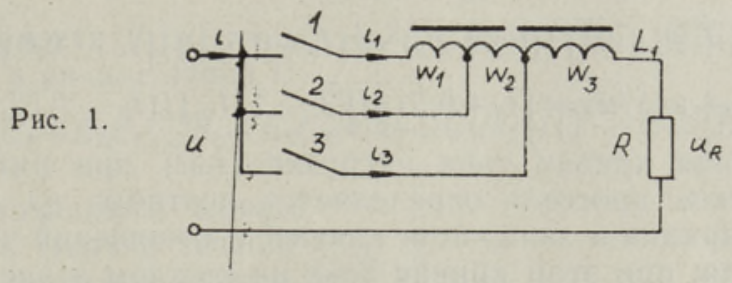

Для упрощения анализа не учитываются рассеяние и активное сопротивление обмотки сглаживающего дросселя, а также падение напряжения на проводящих ключах. Используются следующие обозначения: $u=U_{0}+U_{m} \sin \omega t-$ входное напряжение; $R-$ сопротивление нагрузки; $i, u_{R}$ - ток и напряжение нагрузки; $L_{1}-$ индуктивность сглаживающего дросселя; 1, 2, 3 - полностью управляемые ключи; $i_{1}, i_{2}, i_{3}-$ токи ключей.

Коммутационные функции ключей $1,2,3$ и отношения витков обмотки $w_{1}, w_{2}, w_{3}$ определяются следующим образом. В отпертом состоянии ключа 1 (т. е. при $L_{1}=$ const) ток дросселя и нагрузки имеет вид

$$
i^{\prime}=U_{0} / R+\left[U_{m} / \sqrt{R^{2}+\left(\omega L_{1}\right)^{2}}\right] \sin \left(\theta-\varphi_{1}\right)=I_{0}+I_{m} \sin \left(\theta-\varphi_{1}\right),
$$

где $\varphi_{1}=\operatorname{arctg}\left(\omega L_{1} / R\right)$. Переменная составляющая тока, равная $I_{m} \sin \left(\theta-\varphi_{1}\right)$, аппроксимируется ступенчатой функцией. Параметры ступеней обеспечивают равенство амплитуд и фаз переменной составляющей тока и основной гармоники ступенчатого тока, а также подавление самых низких из высших гармоник последнего. Используя соотношения из [ $\left.{ }^{3}\right]$, получаем, что амплитуда ступеней равна $B_{1}=\pi \sqrt{3} I_{m} / 6$, угол начала ступени $-\alpha-\varphi_{1}=\pi / 6$ и ширина ступени $-2 \pi / 3$. Вычитание из $i^{\prime}(\omega t)$ ступенчатого тока с приведенными параметрами дает расчетную кривую тока $i(\omega t)$, которая содержит гармоники, начиная с пятой (рис. 2).

Зная угловые параметры ступеней, легко определить промежутки, когда ключи 1, 2, 3 находятся в проводящем состоянии: 


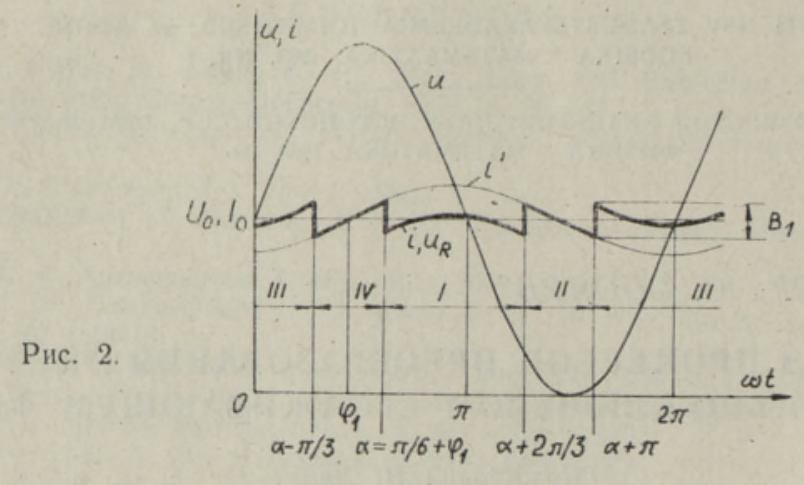

$$
\begin{array}{r}
\alpha+2 \pi n \leqslant \omega t \leqslant \alpha+2 \pi / 3+2 \pi n-\text { ключ } 1 ; \\
\left.\begin{array}{c}
\alpha+2 \pi / 3+2 \pi n \leqslant \omega t \leqslant \alpha+(2 n+1) \pi \\
\alpha+5 \pi / 3+2 \pi n \leqslant \omega t \leqslant \alpha+2 \pi(n+1)
\end{array}\right\}- \text { ключ } 2 ; \\
\alpha+(2 n+1) \pi \leqslant \omega t \leqslant \alpha+5 \pi / 3+2 \pi n-\text { ключ } 3,
\end{array}
$$

где $n=0,1,2,3, \ldots$.

Из вышеприведенных формул для $\left(\alpha-\varphi_{1}\right)$ и $B_{1}$ можно найти отношения витков обмотки дросселя:

$$
\begin{aligned}
& k_{1}=\left(w_{1}+w_{2}+w_{3}\right) /\left(w_{2}+w_{3}\right)=\left(I_{0}+0,5 I_{m}\right) /\left[I_{0}-0,5(\pi / \sqrt{3}-1) I_{m}\right], \\
& k_{2}=\left(w_{2}+w_{3}\right) / w_{3}=\left[I_{0}+0,5(\pi / \sqrt{3}-1) I_{m}\right] /\left(I_{0}-0,5 I_{m}\right) .
\end{aligned}
$$

Расчетная кривая тока нагрузки $i(\omega t)$ при ритмичном изменении числа витков дросселя определяется поэтапно на основе найденных углов отпирания и запирания ключей и отношений числа виткоз обмотки дросселя, при этом кривая тока на каждом этапе описывается классическим уравнением переходного процесса.

Рассмотрим период пульсаций $\alpha \leqslant \omega t \leqslant \alpha+2 \pi$ в установившемся режиме. В промежутке I $\alpha \leqslant \omega t \leqslant \alpha+2 \pi / 3$ отперт. ключ 1 и относительный ток нагрузки изменяется по закону

$$
\begin{gathered}
i_{1}^{*}(\omega t)=1+U_{m}^{*} \cos \varphi_{1} \sin \left(\omega t-\varphi_{1}\right)+ \\
+\left[i_{1}^{*}(\alpha)-1-U_{m}^{*} \cos \varphi_{1} \sin \left(\alpha-\varphi_{1}\right)\right] \exp \left[(\alpha-\omega t) / \omega \tau_{1}\right]
\end{gathered}
$$

где $\tau_{1}=L_{1} / R-$ постоянная времени данной цепи, $\varphi_{1}=\operatorname{arctg} \omega \tau_{1}$, $i_{1}{ }^{*}(\alpha)$ - ток в начале этапа I. За базовые величины примем $U_{\text {баз }}=U_{0}$ и $I_{\text {баз }}=U_{0} / R$.

В промежутке II $\alpha+2 \pi / 3 \leqslant \omega t \leqslant \alpha+\pi$ отперт ключ 2 и относительный ток нагрузки равен

$$
\begin{aligned}
& i_{2}^{*}(\omega t)=1+U_{m}^{*} \cos \varphi_{2} \sin \left(\omega t-\varphi_{2}\right)+\left[i_{2}^{*}(\alpha+2 \pi / 3)-1-\right. \\
& \left.-U_{m}^{*} \cos \varphi_{2} \sin \left(\alpha+2 \pi / 3-\varphi_{2}\right)\right] \exp \left[(\alpha+2 \pi / 3-\omega t) / \omega \tau_{2}\right],
\end{aligned}
$$

где $i_{2}{ }^{*}(\alpha+2 \pi / 3)$ - ток в начале этапа II, $\tau_{2}=\tau_{1} / k_{1}{ }^{2}, \varphi_{2}=\operatorname{arctg} \omega \tau_{2}$.

В промежутке III $\alpha+\pi \leqslant \omega t \leqslant \alpha+5 \pi / 3$ отперт ключ 3 и

$$
\begin{aligned}
& i_{3}^{*}(\omega t)=1+U_{m}^{*} \cos \varphi_{3} \sin \left(\omega t-\varphi_{3}\right)+\left[i_{3}^{*}(\alpha+\pi)-1-\right. \\
& \left.-U_{m}^{*} \cos \varphi_{3} \sin \left(\alpha+\pi-\varphi_{3}\right)\right] \exp \left[(\alpha+\pi-\omega t) / \omega \tau_{3}\right],
\end{aligned}
$$

где $i_{3}{ }^{*}(\alpha+\pi)$ - ток в начале этапа III, $\tau_{3}=\tau_{2} / k_{2}{ }^{2}, \varphi_{3}=\operatorname{arctg} \omega \tau_{3}$.

В промежутке IV $\alpha+5 \pi / 3 \leqslant \omega t \leqslant \alpha+2 \pi$ отперт ключ 2 и 


$$
\begin{aligned}
& i_{4}^{*}(\omega t)=1+U_{m}^{*} \cos \varphi_{2} \sin \left(\omega t-\varphi_{2}\right)+\left[i_{4}^{*}(\alpha+5 \pi / 3)-1-\right. \\
& \left.-U_{m}^{*} \cos \varphi_{2} \sin \left(\alpha+5 \pi / 3-\varphi_{2}\right)\right] \exp \left[(\alpha+5 \pi / 3-\omega t) / \omega \tau_{2}\right],
\end{aligned}
$$

где $i_{4}{ }^{*}(\alpha+5 \pi / 3)$. - ток в начале этапа IV.

Учитывая, что магнитодвижущая сила в дросселе в момент коммутирования не изменяется, запишем значения токов в начале и конце этапов:

$$
\begin{aligned}
& i_{2}^{*}(\alpha+2 \pi / 3)=k_{1} i_{1}^{*}(\alpha+2 \pi / 3)=k_{1}\left[E_{1} i_{1}^{*}(\alpha)+C_{1}\right], \\
& i_{3}^{*}(\alpha+\pi)=k_{2} i_{2}^{*}(\alpha+\pi)=k_{2}\left[E_{2} i_{2}^{*}(\alpha+2 \pi / 3)+C_{2}\right], \\
& i_{4}^{*}(\alpha+5 \pi / 3)=i_{3}^{*}(\alpha+5 \pi / 3) / k_{2}=\left[E_{3} i_{3}^{*}(\alpha+\pi)+C_{3}\right] / k_{2}, \\
& i_{1}^{*}(\alpha)=i_{4}^{*}(\alpha+2 \pi) / k_{1}=\left[E_{2} i_{4}^{*}(\alpha+5 \pi / 3)+C_{4}\right] / k_{1},
\end{aligned}
$$

где $E_{1}=\exp \left(-2 \pi / 3 \omega \tau_{1}\right), \quad E_{2}=\exp \left(-\pi / 3 \omega \tau_{2}\right), \quad E_{3}=\exp \left(-2 \pi / 3 \omega \tau_{3}\right)$;

$$
\begin{aligned}
& C_{1}=1-E_{1}+k_{m} \cos \varphi_{1}\left[\sin \left(\alpha+2 \pi / 3-\varphi_{1}\right)-E_{1} \sin \left(\alpha-\varphi_{1}\right)\right], \\
& C_{2}=1-E_{2}+k_{m} \cos \varphi_{2}\left[\sin \left(\alpha+\pi-\varphi_{2}\right)-E_{2} \sin \left(\alpha+2 \pi / 3-\varphi_{2}\right)\right], \\
& C_{3}=1-E_{3}+k_{m} \cos \varphi_{3}\left[\sin \left(\alpha+5 \pi / 3-\varphi_{3}\right)-E_{3} \sin \left(\alpha+\pi-\varphi_{3}\right)\right], \\
& C_{4}=1-E_{2}+k_{m} \cos \varphi_{2}\left[\sin \left(\alpha-\varphi_{2}\right)-E_{2} \sin \left(\alpha+5 \pi / 3-\varphi_{2}\right)\right] .
\end{aligned}
$$

Условие стационарности $i_{1}(\alpha)=i_{1}(\alpha+2 \pi)$ позволяет найти выражение тока нагрузки в начале этапа I:

$$
i_{1}^{*}(\alpha)=\left[E_{2}^{2} E_{3} C_{1}+E_{2} E_{3} C_{2} / k_{1}+E_{2} C_{3} / k_{1} k_{2}+C_{4} / k_{1}\right] /\left(1-E_{1} E_{2}^{2} E_{3}\right) .
$$

На основе изложенного можно найти кривую тока $i(\omega t)$ в течение всего периода. Для этого подставим выражение (9) в формулу (4) вместо $i_{1}{ }^{*}(\alpha)$ и заменим токи в начале этапов в формулах (5)-(7) найденными таким образом значениями.

Вычисленная по вышеприведенным формулам кривая тока $i$, соответствующая $\omega \tau_{1}=5$, изображена на рис. 3, где показаны и кривая напряжения питания $u$, постоянные составляющие напряжения и тока $U_{0}, I_{0}$, а также напряжения нагрузки $U_{R 0}$. Пунктирные кривые и обозначения, снабженные индексом «прим», соответствуют случаю $L_{1}=$ $=$ const.

Для иллюстрации приведены кривые мгновенных мощностей: $p_{1}=i \cdot u-$ входная мощность, $p_{R}=i \cdot u_{R}-$ мощность нагрузки, $p_{L}=i\left(u-u_{R}\right)-$ мощность дросселя и ключей, а также кривая энергии $W_{L 1}$, накапливаемой дросселем $\left(W_{L 1}=i^{2} L_{1}(t) / 2\right)$.

Рис. 3.

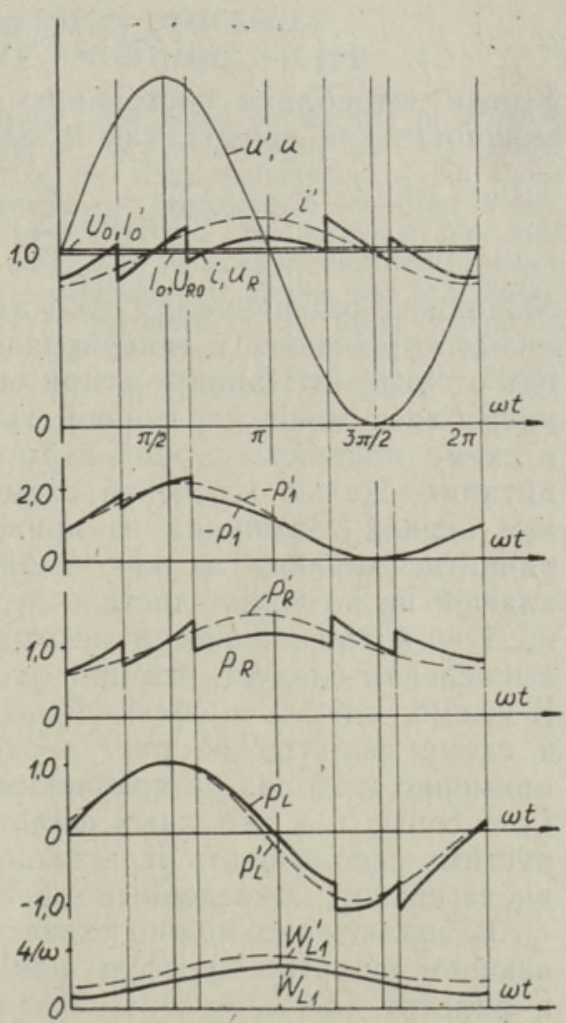


Из рис. 3 видно, что $W_{L 1}<W_{L 1}^{\prime}$, т. е. сглаживающй дроссельь фильтра в случае $L_{1}=$ var накапливает гораздо меньше энергии, чем в случае $L_{1}=$ const.

Используя вычисленную на основе вышеприведенных выражений форму тока, мы нашли мощности элементов исследуемой схемы и проанализировали процессы преобразования в ней энергии. При вычислениях учитывали 60 гармоник в ряде Фурье. Определили постоянную составляющую мощности $P_{(0)}$, мощности первой и второй гармоник $P_{(1)}$ и $P_{(2)}$, а также суммарные мощности четных и нечетных высших гармоник при $\omega \tau_{1}=5,10,20$.

Анализ процессов преобразования энергии показал, что входная мощность содержит постоянную составляющую и мощность первой гармоники

$$
P=P_{(0)}+P_{(1)}
$$

(при $\omega \tau_{1}=10$ отношение $P_{(1)} / P_{(0)}=3,6 \cdot 10^{-3}$ ).

Нагрузка потребляет постоянную составляющую мощности, а также мощность неустраненных первой и высших гармоник

$$
P_{R}=P_{R(0)}+P_{R(1)}+\sum_{n=2}^{\infty} P_{R(n)}
$$

(при $\quad \omega \tau_{1}=10 \quad$ отношения $\quad P_{R(1)} / P_{R(0)}=2,8 \cdot 10^{-4}, \quad P_{R(2)} / P_{R(0)}=2,3 \cdot 10^{-5}$, $\left.\sum_{n=3}^{60} P_{R(n !} / P_{R(0)}=4,5 \cdot 10^{-4}\right)$.

По предположению элементы схемы идеальные, следовательно, $P_{\mathrm{s}}=P_{R}$. Однако $P_{(0)}>P_{R(0)}$ (из рис. 3 видно также, что $U_{0} I_{0}>U_{R 0} I_{0}$, так как $\left.U_{0}>U_{R 0}\right)$. Часть постоянной составляющей входной мощности потребляется ключами

$$
P_{1(0)}+P_{2(0)}+P_{3(0)}=P_{(0)}-P_{R(0)} .
$$

Ключи потребляют постоянную составляющую мощности и генерируют мощность как первой, так и высших гармоник

$$
P_{(0)}-P_{R(0)}=\sum_{n=1}^{\infty} P_{1(n)}+\sum_{n=1}^{\infty} P_{\grave{2}(n)}+\sum_{n=1}^{\infty} P_{3(n)}
$$

Мощность, генерируемая ключами, передается нагрузке. Однако механизм потребления и генерирования мощности первой гармоники зависит от угла $\alpha$. Например, при $\omega \tau_{1}=10\left(\alpha=114^{\circ} 17^{\prime}\right)$ источник питания потребляет мощность первой гармоники. В этом случае генерируемая в схеме мощность первой гармоники передается нагрузке и источнику питания. Целью ключевой схемы фильтра (рис. 1) является устранение первой гармоники из кривой выходного тока, т. е. $P_{R(1)}$ должна равняться нулю. Так как выбранный режим отпирания и запирания ключей не позволил достичь этой цели, то мощности элементов схемы на 1-4-й гармониках в некоторой степени отличаются от нуля. Из вычислений следует, что при $\omega \tau_{1}=10$ отношение $P_{R(1)} / P_{R(0)}=2,8 \cdot 10^{-4}$. В схеме, где $L_{1}=$ const, $P_{R(1)} / P_{R(0)}=4,9 \cdot 10^{-3}$. Отсюда следует, что в схеме фильтра на рис. 1 относительное значение $P_{R(1)}$ уменьшено примерно в 20 раз по сравнению с его значением в схеме фильтра, где $L_{1}=$ const, и в реальных фильтрующих устройствах достаточно и допустимо использовать углы запирания и отпирания ключей и отношения числа витков, приведенные в данной статье.

В заключение можно сказать, что в индуктивно-ключевом сглаживающем фильтре дроссель накапливает гораздо меньше энергии, чем в фильтре, где $L_{1}=$ const. Это позволяет уменьшить массу и габариты 
дросселя. Первая гармоника в выходном токе устраняется не полностью, но ее мощность примерно в 20 раз меньше, чем в схеме фильтра, где $L_{1}=$ const. Ключи исследуемой схемы работают преобразователями частоты, потребляя постоянную составляющую мощности и генерируя мощность как на первой, так и на высших гармониках.

\title{
Л И ТЕ Р А Т У Р А
}

1. Сарв В. В., Сооярв Ю. Э. Авт. свид. СССР № 777782. Бюл. изобр., № 41 (1980).

2. Сооярв Ю. Э. В кн.: Уменьшение искажений в цепях с силовыми полупроводниковыми преобразователями. Таллин, АН ЭССР, 1981, с. 16-19.

3. Липковский К. А., Тонкаль В. Е., Тонкаль С. С. В кн.: Проблемы технической электродинамики, вып. 24. Киев, «Наук. думка», 1970, с. $42-46$.
Ннститут термофизики и электрофизики Академии наук Эстонской ССР
Поступила в редакцию 22 июля 1982

\author{
Maire OJAVEER, J. SOOJARV
}

\section{VENTIIL-INDUKTIIVSES SILUMISFILTRIS TOIMUVATE ENERGEETILISTE MUUNDUSPROTSESSIDE ANALUOS}

On tuletatud valem ventiil-induktiivse silumisfiltri voolu kuju määramiseks ja analüüsitud energia muundamise ja ülekande protsesse selles filtris.

\author{
Maire OJAVEER, J. SOOJARV
}

\section{ANALYSIS OF POWER CONVERSION PROCESSES IN THE SWITCHED-INDUCTANCE SMOOTHING FILTER}

A new circuit of the switched-inductance smoothing filter based on the use of a choke with the inductance varied by stepped switching of its turn number has been elaborated. An expression of the current waveform for the finite inductance of the choke has been derived. The analysis of the power conversion processes in the filter shows that the input power contains power both at zero and at the first harmonic. The load consumes power at zero, the first and higher harmonics. The switches operate as frequency converters consuming power at zero harmonic and generating it at the first and higher harmonics. The power generated in the switches is consumed in the load. Comparing the filter under discussion ( $L=\mathrm{var}$ ) to the one with $L=$ const, the power at the first harmonic is approximately 20 times less and also the stored energy in the choke of the first filter less than in the latter. 